\title{
Study on Pyroelectric Properties of the TGS-TGSe Crystals with Internal Electric Field
}

\author{
J. DZIEDZIC AND J. MRÓZ
}

Institute of Physics, Technical University of Wrocław

Wybrzeże Wyspiańskiego 27, 50-370 Wrocław, Poland

(Received November 22, 2004; revised version June 20, 2005)

\begin{abstract}
Measurements of spontaneous polarization and pyroelectric coefficient versus temperature and electric field were performed for the TGS-TGSe crystals at a definite internal electric field. The obtained results are compared with the thermodynamic theory predictions. The temperature and electric field dependencies of pyroelectric coefficient are presented together with its maximal value as a function of the electric field intensity. It was shown that the temperature, at which the pyroelectric coefficient reaches its maximal value, depends on the external electric field.
\end{abstract}

PACS numbers: $64.70 . \mathrm{Kb}$

\section{Introduction}

Studies on the mixed crystals of triglycine sulphate-triglycine selenate (TGS-TGSe) were initiated by Fatuzzo et al. in paper [1] where, among others, the phase diagram was determined. The detailed investigations of the ferroelectric properties for different compositions were performed by Stankowska [2]. Up to now a series of works appeared related to application of these materials as pyroelectric detectors $[3,4]$. The TGS-TGSe crystals are characterized by an asymmetrical hysteresis loop, which indicates the existence of an internal field. As it was shown by Fatuzzo [5] and Shaulov and Simhony [6], the presence of the internal field affects temperature dependence of pyroelectric coefficient.

The studies on the electric and elastic properties of the pure, as well as the doped with chromium ions, TGS crystals in relation to electric field are presented in papers [7-10]. A detailed analysis of cubic dielectric equation of state in all of three regions corresponding to different types of solution was given [11]. The application of the external electric field in the measurements reduces the space charge effect and allows us to determine its influence. 
The purpose of present paper is to examine the temperature dependence of the pyroelectric coefficient of the $\mathrm{TGS}_{0.1} \mathrm{Se}_{0.9}$ and $\mathrm{TGS}_{0.7} \mathrm{Se}_{0.3}$ crystals, from which the space charge was removed.

\section{Thermodynamics relations}

The pyroelectric coefficient is defined as the temperature derivative of polarization at a constant deformation

$$
\gamma=\left(\frac{\partial \boldsymbol{P}}{\partial \boldsymbol{T}}\right)_{\eta}
$$

Replacing the electric field in the Gibbs potential the sum

$$
E_{x}=E+E_{b}
$$

where $E$ - external electric field, $E_{b}$ - internal electric field, and performing the corresponding transformations described in paper [10] the relationship for $\gamma$ was obtained:

1. In the ferroelectric phase $T<T_{\mathrm{c}}$ and $p<0$ for $\left|E_{b}+E\right| \leq\left|E_{\mathrm{M}}\right|$

$$
\gamma_{1}=\frac{\sqrt{-A}}{\varepsilon_{0} C A \sqrt{3 B}} \cos \left(\frac{\varphi}{3}\right)-\frac{3\left(E_{b}+E\right) \sqrt{3 B}}{2 \varepsilon_{0} C A^{2} \sqrt{1+\frac{27 B\left(E_{b}+E\right)^{2}}{4 A^{3}}}} \sin \left(\frac{\varphi}{3}\right),
$$

where

$$
A=\frac{T-T_{\mathrm{c}}}{\varepsilon_{0} C}, \quad \varphi=\arccos \left[-\frac{3\left(E+E_{b}\right)}{2 A} \sqrt{-\frac{A}{3 B}}\right], \quad E_{M}=\sqrt{-\frac{4 A^{3}}{27 B}} .
$$

2. For $T<T_{\mathrm{c}}$ and $\left|E+E_{b}\right| \geq\left|E_{\mathrm{M}}\right|$ in the ferroelectric phase

$$
\gamma_{2}=\frac{A^{2}}{54 \varepsilon_{0} C B^{3} \sqrt{\frac{\left(E_{b}+E\right)^{2}}{4 B^{2}}+\frac{A^{3}}{27 B^{3}}}}\left[\frac{1}{\sqrt[3]{\left(\frac{E_{b}+E}{2 B}+d\right)^{2}}}+\frac{1}{\sqrt[3]{\left(\frac{E_{b}+E}{2 B}-d\right)^{2}}}\right],
$$

where $d=\sqrt{\frac{\left(E+E_{b}\right)^{2}}{4 B^{2}}+\frac{A^{3}}{27 B^{3}}}$.

3. For $T>T_{\mathrm{c}}, p>0$ and $E+E_{b} \neq 0$ paraelectric phase

$$
\gamma_{3}=\frac{A^{2}}{54 \varepsilon_{0} C B^{3} \sqrt{\frac{\left(E_{b}+E\right)^{2}}{4 B^{2}}+\frac{A^{3}}{27 B^{3}}}}\left[\frac{1}{\sqrt[3]{\left(\frac{E_{b}+E}{2 B}+d\right)^{2}}}+\frac{1}{\sqrt[3]{\left(\frac{E_{b}+E}{2 B}-d\right)^{2}}}\right] .
$$

A shift of temperature, at which $\gamma$ reaches maximum, as a function of the total field was also determined

$$
\Delta T=\frac{3 B^{1 / 3}}{2^{4 / 3} A}\left(E+E_{b}\right)^{2 / 3}
$$

\section{Experimental}

The phase transition temperature, the coefficients of the thermodynamic potential, and the Curie-Weiss constants were determined on the basis of the measurement results. 
TABLE

Thermodynamic constants for the (TGS) $x$ (TGSe) $)_{1-x}$ crystals.

\begin{tabular}{c|c|c|c|c}
\hline \hline$x$ & $B\left[\mathrm{~V} \mathrm{~m}^{5} \mathrm{C}^{3}\right]$ & $T_{\mathrm{c}}[\mathrm{K}]$ & $E_{b}[\mathrm{~V} / \mathrm{m}]$ & $C[\mathrm{~K}]$ \\
\hline 0.1 & $2.5 \times 10^{12}$ & 304.86 & 4075 & 4500 \\
0.7 & & 319.82 & 5737 &
\end{tabular}

The temperature and electric field dependencies of the pyroelectric coefficient were calculated using data collected in Table and the thermodynamic relations. The results of the calculations are illustrated in Figs. 1 and 2.

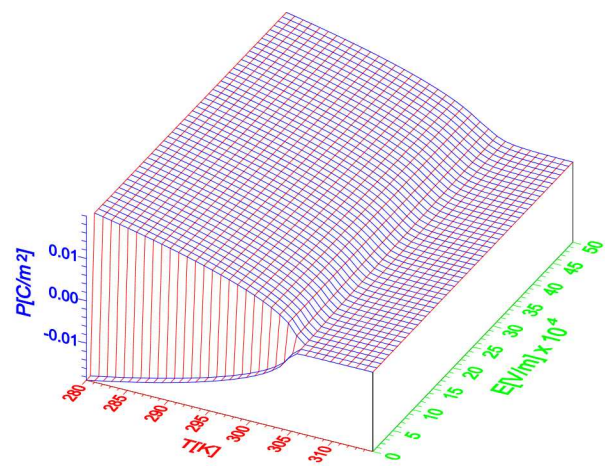

Fig. 1. Theoretical dependence of the spontaneous polarization versus temperature and biasing electric field for the TGS-TGSe crystals.

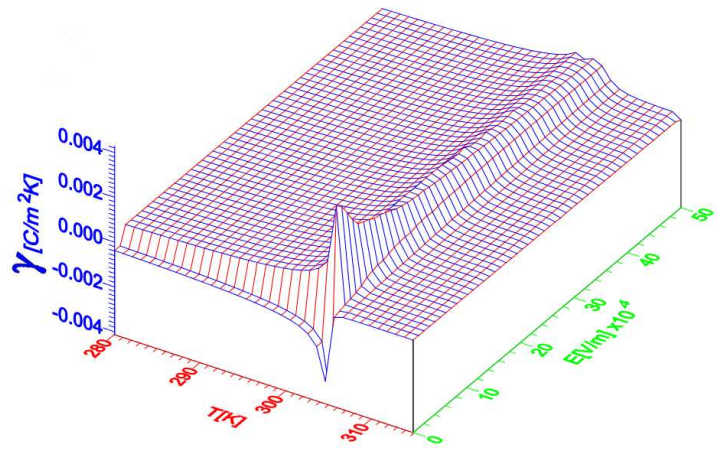

Fig. 2. Theoretical dependence of the pyroelectric coefficient versus temperature and biasing electric field for the TGS-TGSe crystals.

The investigations of the spontaneous polarization and pyroelectric coefficient versus temperature were performed by means of the pyroelectric charge integration method using the system described in paper [9]. 
The measurements were carried out for the $\mathrm{TGS}_{0.1} \mathrm{Se}_{0.9}$ and $\mathrm{TGS}_{0.7} \mathrm{Se}_{0.3}$ crystals grown up in the ferroelectric phase. The composition of the crystals $(x=0.1$ and 0.7$)$ was determined on the basis of the phase diagram [1].

Samples for measurements were cut in a shape of discs with the flat surface perpendicular to the ferroelectric axis. Gold electrodes were evaporated on this surface perpendicular to ferroelectric axis. The cuprum wires were stick by silver paste to these electrodes. These wires were a suspension for the samples in thermostat and simultaneously they play a role of the electrical contacts. The samples prepared in this way were located in thermostat and were annealed at temperature $360 \mathrm{~K}$ during 18 hours. Directly after annealing the measurements were performed on the crystals during cooling scans. The temperature rate was $c a .0 .02 \mathrm{~K} / \mathrm{s}$.

\section{Results}

The measurements of the ferroelectric hysteresis loop were done at temperature $278 \mathrm{~K}$ at low frequency electric field by means of the electric charge integration method. The rate of the change of the electric field was equal to $600 \mathrm{~V} /(\mathrm{m} \mathrm{s})$. The shape of the hysteresis loop is shown in Fig. 3.

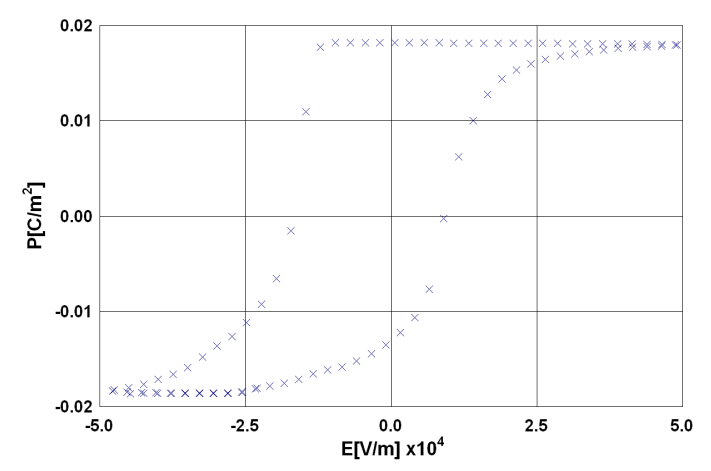

Fig. 3. The hysteresis loop measured in a slow-changing electric field.

From the loop the value of the internal electric field intensity was determined, which was equal to $4075 \mathrm{~V} / \mathrm{m}$ (a shift of the center of the hysteresis loop).

From the measurements of the hysteresis loop the temperature dependence of the internal field for the $\mathrm{TGS}_{0.1} \mathrm{Se}_{0.9}$ and $\mathrm{TGS}_{0.7} \mathrm{Se}_{0.3}$ crystals was determined. The dependence of $E_{b}$ versus temperature is shown in Fig. 4. It should be noticed that $E_{b}$ diminishes to zero at temperature $T_{\mathrm{c}}$.

The investigations of the pyroelectric coefficients were carried out for the $\mathrm{TGS}_{0.1} \mathrm{Se}_{0.9}$ and $\mathrm{TGS}_{0.7} \mathrm{Se}_{0.3}$ crystals as a function of temperature for different values of electric field biasing the crystals. The experimental data were elaborated by the cringing method and they are shown in the $3 \mathrm{D}$ system using the $T-E$ grid.

The results of the polarization studies are presented in Fig. 5. The external field was applied in the opposite direction with respect to the internal field. This 


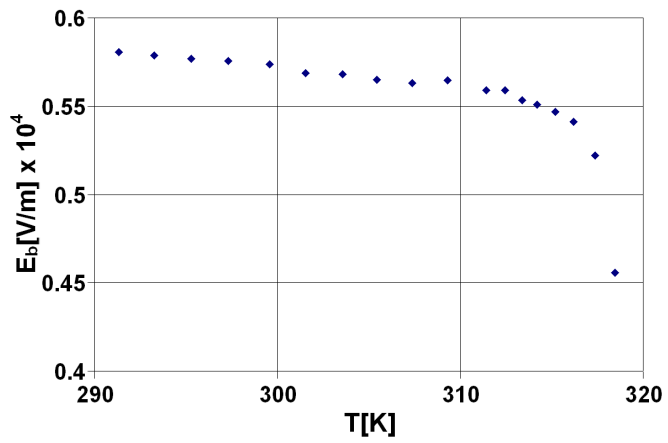

Fig. 4. The dependence of internal field versus temperature for the $\mathrm{TGS}_{0.7} \mathrm{Se}_{0.3}$ crystals.
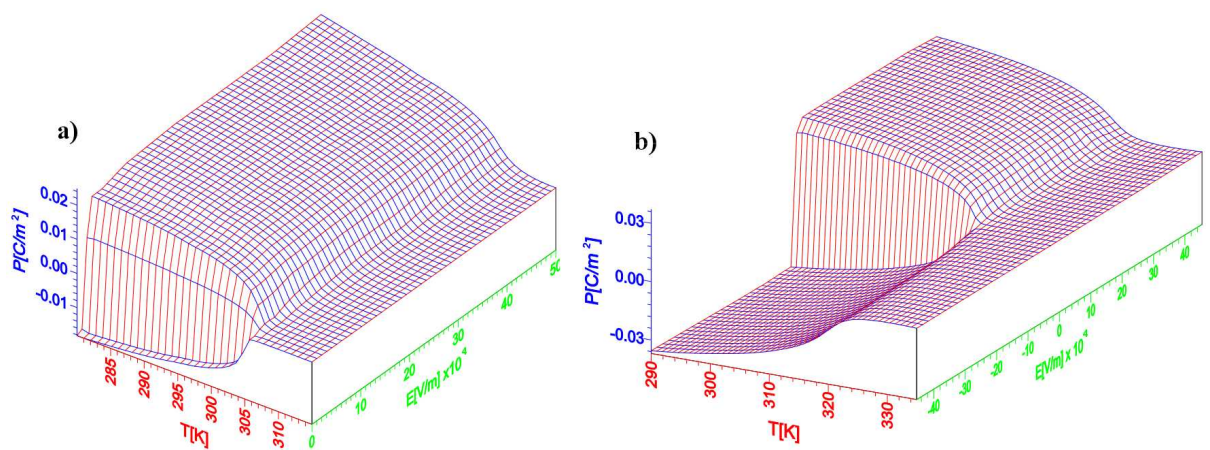

Fig. 5. The dependence of polarization versus temperature and electric field for the $\operatorname{TGS}_{x} \mathrm{Se}_{1-x}$ : (a) $x=0.1$, (b) $x=0.7$.

caused the compensation of the internal field when the external field, equal in value but in opposite direction, was applied. If the internal value of electric field is crossed, the direction of the polarization changes.

The results of measurements confirmed the predictions resulting from the thermodynamic theory. The electric field causes a change in the temperature dependence of polarization. Moreover, the changes are the greater the closer the temperature is to the phase transition temperature. In all cases electric field causes the diffusion of the phase transition, the diffusion rises with the increase in biasing electric field. The numerical values of the polarization at temperature $280 \mathrm{~K}$ obtained in the measurements are consistent with the values obtained from the theoretical calculation. The character of the changes with either temperature or electric field is similar in a case of both theoretical and experimental dependencies. The results of measurements of the pyroelectric coefficient are presented in Fig. 6 .

The values of the pyroelectric coefficient were calculated using a method of differentiation of the $P=f(T)$ dependence. When the biasing field crosses the $E_{b}$ values, the pyroelectric coefficient changes its sign. Along with the increase in 

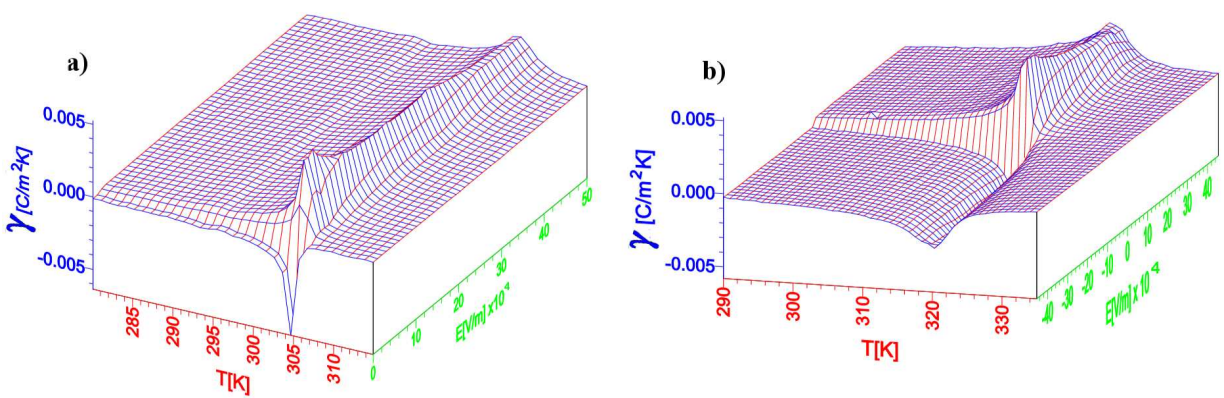

Fig. 6. The dependence of the pyroelectric coefficient versus temperature and electric field for the $\mathrm{TGS}_{x} \mathrm{Se}_{1-x}$ : (a) $x=0.1$, (b) $x=0.7$.

electric field the diffusion of the pyroelectric coefficient and a shift of its maximum value towards high temperature follows from the observation.

The effect of the electric field on the maximum values of the pyroelectric coefficient is shown in Fig. 7.

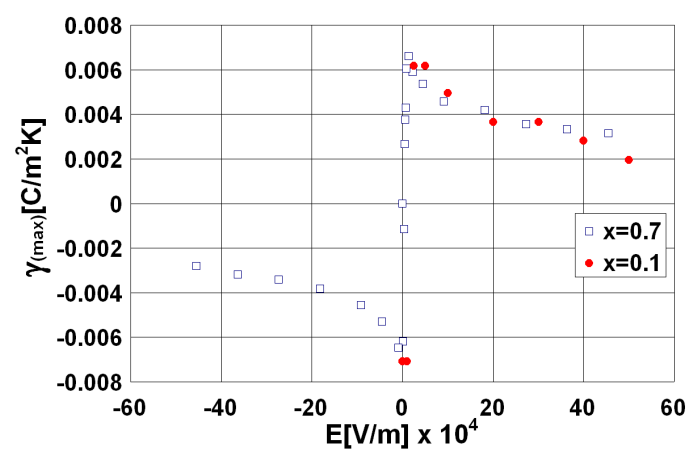

Fig. 7. The dependence of the peak value of the pyroelectric coefficient versus external electric field for the $\mathrm{TGS}_{x} \mathrm{Se}_{1-x}$ crystals.

We may observe in Fig. 7 the insignificant differences of the $\gamma$ value at the sign change of the biasing field for both compositions as well as the different shift on the $E$ axis.

The shift in temperature $T_{\mathrm{m}}-T_{\mathrm{c}}$ (the $\gamma_{\max }$ shift) as a function of electric field is shown in Fig. 8. When this relationship is presented in the coordination system $\Delta T=f\left(E^{2 / 3}\right)$ (Fig. 8), a confirmation of the equation $\Delta T=\alpha E^{2 / 3}$ is obtained.

The region broadens towards high temperature with the increase in electric field. After crossing the Curie temperature the pyroelectric temperature does not become at once equal to zero but gradually decreases (as the mass of paraelectric region significantly surpasses the ferroelectric one). If the electric field intensity increases, $\gamma_{\max }$ decreases, simultaneously the width of the $\gamma=f(T)$ curve becomes 


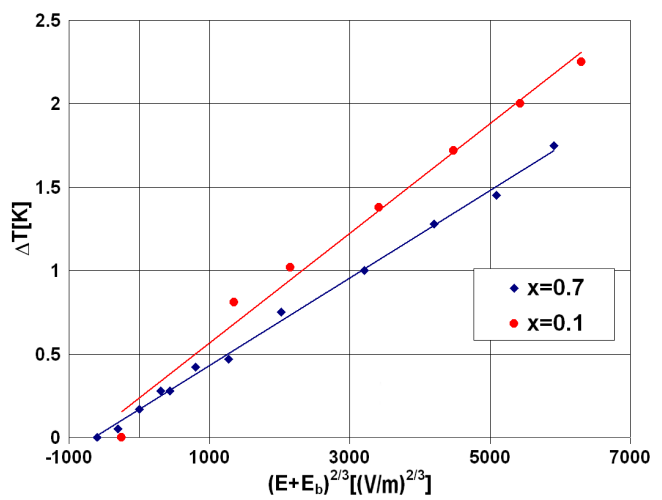

Fig. 8. Dependence of temperature at which $\gamma$ reaches maximum, $\Delta T$ versus $\left(E+E_{b}\right)^{2 / 3}$.

larger. A shift of temperature at which pyroelectric coefficient reaches maximum is simultaneously observed (Fig. 8). The shift is consistent with the equation given in the paper [9].

The qualitative and quantitative character of the changes in the pyroelectric coefficient versus either temperature or electric field, obtained as a result of the experiment, agrees with the theoretical one.

\section{Discussion}

The results of the studies confirmed the dependencies, predicted by the thermodynamic theory, of both the spontaneous polarization and pyroelectric coefficient versus temperature and biasing electric field. This compatibility is not only qualitative but also, within sufficient accuracy, quantitative.

Electric field can identify the given direction of the vibrations in the crystal lattice leading to a break of the equipartition energy theorem on each degree of freedom. This effect becomes more intense along with an increase in the electric field intensity. This causes a perturbation in the statistical distribution of the ions vibrations in the crystal lattice, which in turn causes the diffusion of the phase transition as well as the shift of its temperature towards high temperature.

All dependencies $\gamma=f(T)$ possess clear maxima at temperature close to the phase transition one. These maxima are connected with fast disappearance of polarization at the phase transformation in the crystal.

The maximum value of the pyroelectric coefficient is the biggest, as regards to its absolute value, for the external field equal to the internal one and then it diminishes with the external field increase (Fig. 7). Simultaneously the electric field causes the shift of the temperature, at which $\gamma$ reaches maximum value, towards high temperature (Fig. 8).

The investigations revealed that in the ferroelectric phase at temperatures far from $T_{\mathrm{c}}$ the pyroelectric coefficient changes its sign at the electric field slightly 
larger than the internal one as well as its value rises with increase in electric field intensity reaching a saturation state. However, there exists a clear dependence between these magnitudes in the vicinity of the phase transition (Fig. 8). It can be seen in the figure that the sign of the pyroelectric coefficient changes with the increase in the electric field. The change of the sign follows at the $E$ values greater than $E_{\mathrm{b}}$ (electric field $E$ is directed opposite to $E_{b}$ ).

The $\mathrm{TGS}_{x} \mathrm{Se}_{1-x}$ crystals, grown up in the ferroelectric phase, are characterized by the asymmetric hysteresis loop obtained at the low frequency field and at the internal field determined by the shift of the loop. The $E_{b}$ field does not diminish to zero at phase transition as well as at the annealing. It has a permanent character. From the $\gamma$ measurements versus temperature it results that the pyroelectric coefficient reaches a maximum value at $E_{z}=-E_{b}$, which indicates that the crystals, grown up in the ferroelectric phase, are in the monodomain state. The values of the coefficients $\alpha=\frac{3 B^{1 / 3}}{2^{4 / 3} A}$ from Eq. (6) determined from the slopes of the $\Delta T=\alpha\left(E+E_{b}\right)^{2 / 3}$ lines are equal to:

- for $x=0.1, \alpha_{1}=0.00033$,

- for $x=0.7, \alpha_{2}=0.00026$.

In the case of the pyroelectric properties the internal field plays a positive role stabilizing the domain structure.

\section{References}

[1] E. Fatuzzo, R. Nitsshe, Z. Electrochem. 62, 970 (1959).

[2] J. Stankowska, Fizyka Dielektryków i Radiosp. 4, 151 (1968).

[3] P.W. Whipps, K.L. Bye, Ferroelectrics 7, 183 (1974).

[4] K.L. Byc, P.W. Whipps, E.T. Keve, Ferroelectrics 7, 179 (1974).

[5] E. Fatuzzo, J. Appl. Phys. 31, 1029 (1960).

[6] A. Shaulov, M. Simhony, J. Appl. Phys. 47, 1 (1976).

[7] J. Dziedzic, Ph.D. thesis, Technical University of Wrocław, Wrocław 1979.

[8] J. Dziedzic, H. Wolniewicz, Mater. Sci. 18, 41 (1992).

[9] J. Dziedzic, B. Stolecki, Acta Phys. Pol. A 98, 55 (2000).

[10] J. Dziedzic, Acta Phys. Pol. A 98, 373 (2000).

[11] V.B. Samoylov, Y.S. Yoon, Ferroelectrics 263, 11 (2001). 